\title{
Avaliação Externa de Escolas: Perspetivas dos Diretores da Região Norte de
}

\section{Portugal}

\section{External School Evaluation: Perspectives of principals from the Northern Region of Portugal}

\author{
Filipa Seabra*, José Augusto Pacheco** \\ *Universidade Aberta (Portugal), CIEd-UMinho, LE@D, **Universidade do Minho (Portugal), CIEd-UMinho
}

\begin{abstract}
Resumo
Este trabalho insere-se no âmbito do Projeto de investigação «Impacto e Efeitos da Avaliação Externa nas Escolas do Ensino Não-Superior» (FCT PTDC/CPE-CED/116674/2010), desenvolvido por 6 universidades nacionais. Em concreto, situa a sua análise sobre os resultados de um inquérito por questionário enviado a todos os diretores de unidades educativas do continente português. Analisamos, neste texto, os resultados atinentes às perspetivas da Região Norte de Portugal, por ser aquela que está geograficamente mais próxima da Universidade do Minho. Os resultados apontam para uma perceção moderadamente positiva da Avaliação Externa de Escolas.

Palavras-chave: [Avaliação externa de escolas, Diretores de escolas, Impacto e efeitos].
\end{abstract}

\begin{abstract}
This paper is a part of the project «Impact and Effects of External Evaluation of Non-Higher Education Schools» (FCT PTDC/CPE-CED/116674/2010), undertaken by six Portuguese universities. It focuses on the results of a survey by questionnaire sent to all principals of schools in the Portuguese mainland. In this text, we analyse results concerning the perspectives of principles from the northern region of Portugal, since it is geographically closest to the University of Minho. The results point to a moderately positive appraisal of External School Evaluation.

Keywords [External school evaluation, School principals, Impact and effects].
\end{abstract}

\section{Introdução}

A Avaliação Externa das Escolas públicas do ensino não superior tem vindo a ser desenvolvida, na atual modalidade, pela Inspeção Geral de Educação e Ciência (IGEC) desde 2005. Findo um primeiro ciclo de avaliação, e iniciado um segundo, em 2011, impunha-se levar a cabo processos de investigação sobre esta realidade, com vista a conhecer os seus impactos e efeitos sobre as práticas dos diversos agentes educativos implicados nesse processo. Foi desse desiderato que teve origem o projeto de investigação «Impacto e Efeitos da Avaliação Externa nas Escolas do Ensino não Superior» (AEEENS), (PTDC/CPE-CED/116674/2010), no qual se insere a presente comunicação, e que contou com a participação de seis universidades portuguesas, mapeando a realidade do continente português.

No âmbito deste projeto, foi elaborado um questionário que foi enviado a todos os diretores de unidades educativas abrangidas pelo processo de avaliação externa e que permite aos inquiridos, enquanto interlocutores privilegiados pelo conhecimento que detêm das escolas que dirigem, exprimir as suas opiniões sobre os seguintes aspetos: a) domínios e facetas dos efeitos da avaliação externa de escolas; b) Loci (impacto sobre diferentes elementos da escola); c) fatores potenciadores e inibidores do impacto da avaliação externa; d) avaliação global do processo de avaliação externa e e) eficiência do processo de avaliação externa.

$\mathrm{Na}$ presente comunicação, analisamos os principais resultados deste inquérito, focalizando as perspetivas dos Diretores de escolas da região Norte de Portugal.

\section{A Avaliação Externa de Escolas (AEE) no contexto português: impactos e efeitos}

A avaliação institucional é um processo de grande complexidade, impossibilitando análises controladas e uma mensuração linear de resultados e apelando a processos profundos, inseridos no contexto real de cada escola (Pacheco, Seabra \& Morgado, 2014).

Os objetivos atualmente elencados para a AEE prendem-se com a melhoria dos resultados e aprendizagens dos alunos, a responsabilização, a participação da comunidade local na escola e a regulação da educação (IGEC, 2011).

A AEE tem sido reconhecida internacionalmente como detendo a potencialidade de promover mudanças ao nível das políticas educativas, bem como aos níveis organizacional, curricular e pedagógico (Pacheco, Seabra \& Morgado, 2014). Pode, no entanto, promover reações por parte das escolas, como sejam a «visão em túnel» (foco nos aspetos quantitativos, sobretudo os resultados académicos); a «miopia» (medidas orientadas para o curto prazo); a «ossificação» (mudança da escola para corresponder ao recomendado, de forma rígida), e «fixação na medida» (foco no resultado, mais do que no processo de melhoria), para as quais é necessário estar alerta (Ehren \& Visscher, 2006).

$O$ projeto AEEENS caracteriza-se pela sua abrangência nacional e pluralidade metodológica (Moreira \& Rodrigues, 2015), no entanto, e na medida em que "embora a AEE siga um modelo que impõe 
normas uniformes e tendencialmente conducentes a uma homogeneização de procedimentos organizacionais”, a forma como o processo é percecionado pelos atores escolares em diferentes contextos tem revelado discrepâncias relevantes, "que sublinham a importância do contexto" (Rodrigues, Seabra, Queirós, Sousa, Lamela, Costa \& Morgado, 2015, no prelo, s.p.), justifica-se a realização e apresentação de análises parcelares, que procurem salientar eventuais disparidades entre contextos locais, bem como aspetos transversais a nível nacional. É nesse contexto que se situam, não só os estudos de caso realizados no âmbito do projeto, mas também a análise parcelar dos questionários a diretores que apresentamos neste texto, e que se foca sobre a região do país mais próxima da Universidade do Minho.

\section{Metodologia}

A metodologia empregue para a análise apresentada no presente texto foi inteiramente quantitativa (Almeida \& Freire, 2008; Moreira, 2007; Tuckman, 2000). Visamos assim a apresentação de uma perspetiva ampla e tão representativa quanto possível das perspetivas dos diretores de unidades educativas localizadas na região Norte do país sobre o processo de AEE.

Outros elementos fundamentais para a compreensão dos impactos e efeitos da AEE nas escolas do ensino superior foram recolhidos através de outras metodologias, entre as quais destacamos a análise documental e a realização de estudos de caso, contemplando entrevistas aprofundadas a diversos elementos da comunidade educativa. Assim, os dados apresentados nesta comunicação devem ser entendidos como uma análise parcial de uma realidade complexa; um dos ângulos de análise que deve ser perspetivado em relação com outros ângulos, vários dos quais tidos em conta no projeto.

O questionário aplicado foi elaborado por elementos do Projeto AEEENS (Rodrigues \& Moreira, 2015), e sujeito a diversos procedimentos de aperfeiçoamento e pré-teste antes do seu envio por e-mail a todos os diretores de escolas do território de Portugal continental, isto é, ao universo em estudo. O instrumento inclui, além de dados de caracterização do respondente e da unidade orgânica, as seguintes partes: a) domínios e facetas dos efeitos da avaliação externa de escolas; b) Loci (impacto sobre diferentes elementos da escola); c) fatores potenciadores e inibidores do impacto da avaliação externa; d) avaliação global do processo de avaliação externa e e) eficiência do processo de avaliação externa.

As análises estatísticas realizadas, de natureza descritiva, recorreram ao SPSS v. 21.

\section{Caracterização da amostra}

A amostra recolhida corresponde à taxa de resposta obtida, tratando-se assim de uma amostragem não intencional.
Responderam ao questionário 52 Diretores de unidades educativas da Região Norte de Portugal. Estes diretores integram o Agrupamento/Escola em questão há uma média de 15,1 anos (DP = 8,31), assumem cargos de direção, em média, há 10,7 anos (DP = 6,8) registam-se, no entanto, fortes dispersões nestas medidas. Assumiam funções diretivas aquando da mais recente avaliação externa 41 desses diretores (80,4\%). Quarenta e dois (82,4\%) tiveram formação específica em administração, liderança ou gestão escolar.

As escolas que dirigem são, na sua maioria, agrupamentos de escolas de Ensino Básico e Pré-Escolar; apenas 19,2\% incluem uma ou mais escolas secundárias.

80,8\% das unidades orgânicas foram avaliadas no $1 .^{\circ}$ ciclo de AEE, com maior incidência nos anos de 2007/08 e 2008/09 (cada um dos quais com 30,8\%). No segundo ciclo de AEE, 32 unidades orgânicas foram avaliadas $(61,5 \%)$ - estes valores podem ser explicados pelo facto de ter ocorrido durante este processo uma reorganização da rede escolar. Nos casos em que as unidades orgânicas sofreram fusões ou reorganizações após a avaliação, pediu-se que indicassem não terem sido avaliadas.

Das escolas avaliadas no 2. ${ }^{\circ}$ ciclo de AEE, 8 foram-no em 2011/12, 6 em 2012/13 e 10 em 2013/14.

Os Diretores avaliam de forma muito variada a estabilidade do seu corpo docente, registando-se elevados desvios padrão em todas as medidas que permitem aferir este aspeto (ex.: percentagem de docentes em Quadro de Agrupamento).

\section{Resultados}

Quanto à avaliação recebida nesse processo de AEE (Ver figura 1), a classificação mais frequente no domínio Resultados foi Bom ( $\mathrm{n}=24,57,1 \%)$, seguida de Suficiente $(n=14,33,3 \%$ de respostas válidas). Houve apenas uma classificação de Insuficiente e 3 de Muito Bom.

No domínio Prestação do Serviço Educativo, registou-se uma maioria de avaliações de Bom $(n=31$, $73,8 \%$ ), embora tenha havido 7 classificações de suficiente (16,7\%) e 4 de Muito Bom $(9,5 \%$ de percentagem válida).

No domínio da Organização e Gestão Escolar, as classificações foram, em ambos os casos, o Bom (respetivamente $n=19,46,3 \%$ e $n=18,43,9 \%$ de percentagem válida), seguindo-se de perto o Muito Bom (respetivamente $n=16,39,0 \%$ e $n=15,36,6 \%$ de percentagem válida), pelo que estes dois domínios se destacam como os melhor avaliados no primeiro ciclo da AEE, para este grupo de escolas, seguindo de perto a tendência nacional.

Já no que diz respeito à capacidade de autorregulação e melhoria, este foi o domínio com apreciação menos positiva, com 2 insuficientes (5\%), 16 suficientes (40\%), 20 bons (50\%) e 2 Muito Bons (5\% das respostas válidas). 


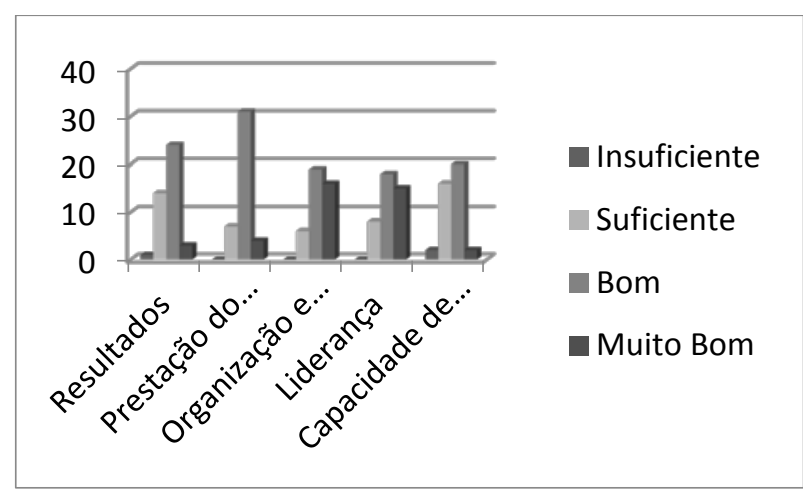

Figura 1 - Resultados nos 5 domínios da Avaliação Externa obtidos pelas unidades orgânicas no $1 .^{\circ}$ ciclo de AEE.

No 2. ${ }^{\circ}$ ciclo de AEE, e após a reformulação do modelo que a orienta, passaram a ser alvo de avaliação 3 domínios, que vieram reorganizar os 5 anteriores. Também a escala de avaliação se alterou, passando a englobar mais um nível, o Excelente. Uma vez que os dados começaram a ser recolhidos durante esse ciclo, algumas unidades orgânicas ainda não conheciam os resultados da avaliação a que tinham sido sujeitas.

Quanto às classificações obtidas pelas escolas incluídas na amostra (Figura 2), os extremos da escala (Insuficiente e Excelente) estiveram ausentes. Os resultados mais positivos verificaram-se no domínio da Liderança e Gestão, seguido do da Prestação do Serviço Educativo, e por último pelo dos Resultados.

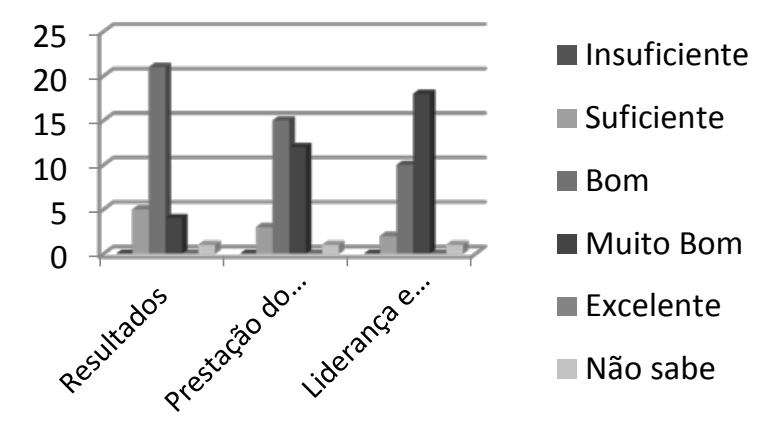

Figura 2 - Resultados nos 3 domínios da Avaliação Externa obtidos pelas unidades orgânicas no $2{ }^{\circ}$ ciclo de AEE.

\section{Domínios e facetas dos efeitos da avaliação externa de escolas}

Relativamente aos domínios e facetas dos efeitos da AEE, pediu-se aos respondentes que se posicionassem, numa escala de 7 pontos, entre -3 (extremamente negativo) e +3 (extremamente positivo) quanto ao peso dos efeitos da AEE em diversos aspetos particulares, organizados de acordo com os domínios de avaliação.

Apresentam-se, de seguida, os 5 contextos em que o diretor avaliou o efeito da AEE como mais positivo, e menos positivo, em cada um dos domínios da AEE (Tabelas 1 a 3).

Relativamente ao domínio Resultados, os diretores avaliaram como moderadamente positivo o impacto da AEE em todos os itens. Avaliaram mais positivamente itens relacionados com a relação entre a escola e a comunidade e com a avaliação interna dos resultados dos alunos, e menos positivamente aspetos mais diretamente relacionados com os alunos e com a avaliação externa das aprendizagens (Tabela 1)

Tabela 1.

Domínios e facetas dos efeitos da AEE - Domínio Resultados.

\begin{tabular}{|c|c|c|}
\hline Itens & Média & DP \\
\hline $\begin{array}{l}\text { Valorização da escola } \\
\text { comunidade envolvente }\end{array}$ & 1,37 & 1,268 \\
\hline $\begin{array}{l}\text { Contributo da escola para o } \\
\text { desenvolvimento } \\
\text { envolvente }\end{array}$ & 1,35 & 1,235 \\
\hline $\begin{array}{l}\text { Participação dos professores na vida } \\
\text { da escola }\end{array}$ & 1,17 & 1,115 \\
\hline Relacionamento escola-família & 1,17 & 1,279 \\
\hline $\begin{array}{l}\text { Resultados académicos avaliados } \\
\text { internamente (p. ex., notas, transições) } \\
(. . .)\end{array}$ & 1,15 & 1,195 \\
\hline $\begin{array}{l}\text { Comportamento e disciplina dos } \\
\text { alunos }\end{array}$ & 0,87 & 1,237 \\
\hline $\begin{array}{l}\text { Aprendizagens reais por parte dos } \\
\text { alunos }\end{array}$ & 0,83 & 1,115 \\
\hline $\begin{array}{l}\text { Resultados académicos avaliados } \\
\text { externamente (p. ex., exames) }\end{array}$ & 0,81 & 1,189 \\
\hline Taxas de abandono e desistência & 0,81 & 1,496 \\
\hline $\begin{array}{l}\text { Solidariedade e relacionamento } \\
\text { entre os alunos }\end{array}$ & 0,77 & 1,277 \\
\hline
\end{tabular}

Relativamente ao domínio Prestação do Serviço Educativo, volta a registar-se uma avaliação moderadamente positiva do impacto da AEE em todos os itens, destacando-se positivamente aspetos relacionados com a gestão curricular, monitorização, partilha entre docentes e promoção do sucesso, e menos positivamente o impacto sobre o uso dos recursos, a promoção da equidade e diferenciação curriculares, a valorização das dimensões artística, ética e/ou cultural e a contextualização do currículo (Tabela 2).

Tabela 2.

Domínios e facetas dos efeitos da AEE - Domínio Prestação do Serviço Educativo.

\begin{tabular}{|c|c|c|}
\hline Itens & Média & SD \\
\hline $\begin{array}{l}\text { Adoção de medidas de promoção do } \\
\text { sucesso escolar }\end{array}$ & 1,50 & 1,229 \\
\hline $\begin{array}{l}\text { Articulação na gestão do currículo } \\
\text { (p. ex., entre professores, } \\
\text { departamentos, níveis) }\end{array}$ & 1,40 & 1,107 \\
\hline $\begin{array}{l}\text { Monitorização interna do } \\
\text { desenvolvimento do currículo (p. ex., } \\
\text { coordenação das planificações e } \\
\text { avaliações) }\end{array}$ & 1,35 & 1,219 \\
\hline $\begin{array}{l}\text { Partilha de conhecimentos } \\
\text { experiências pedagógicas entre } \\
\text { professores }\end{array}$ & 1,31 & 1,130 \\
\hline $\begin{array}{l}\text { Monitorização e adoção de medidas } \\
\text { de prevenção da desistência e } \\
\text { abandono } \\
\text { (...) }\end{array}$ & 1,31 & 1,261 \\
\hline $\begin{array}{l}\text { Eficiência no uso do tempo e dos } \\
\text { recursos educativos }\end{array}$ & 0,96 & 1,137 \\
\hline
\end{tabular}




\begin{tabular}{|c|c|c|}
\hline $\begin{array}{l}\text { Ações de promoção da equidade e } \\
\text { justiça curricular }\end{array}$ & 0,93 & 1,253 \\
\hline $\begin{array}{l}\text { Contextualização do currículo e } \\
\text { abertura ao meio }\end{array}$ & 0,90 & 1,159 \\
\hline $\begin{array}{l}\text { Adequação do trabalho educativo } \\
\text { aos ritmos de aprendizagem dos alunos }\end{array}$ & 0,90 & 1,192 \\
\hline $\begin{array}{l}\text { Valorização das dimensões artística, } \\
\text { ética e/ou cultural no ensino }\end{array}$ & 0,83 & 1,133 \\
\hline
\end{tabular}

Por fim, relativamente ao domínio Liderança e Gestão, registar-se uma avaliação moderadamente positiva do impacto da AEE em todos os itens embora um pouco mais positiva do que nos outros domínios. Destacam-se expressivamente vários aspetos relacionados com a autoavaliação e melhoria, e são menos expressivos os aspetos que se relacionam com o desempenho e desenvolvimento profissional de professores e funcionários, a comunicação externa e o acompanhamento do percurso pós-escolar dos alunos (Tabela 3).

Tabela 3.

Domínios e facetas dos efeitos da AEE - Liderança e Gestão

\begin{tabular}{|c|c|c|}
\hline Itens & Média & SD \\
\hline $\begin{array}{c}\text { Qualidade dos processos de } \\
\text { autoavaliação da escola/agrupamento }\end{array}$ & 1,62 & 1,27 \\
\hline $\begin{array}{l}\text { Valorização dos processos de } \\
\text { autoavaliação }\end{array}$ & 1,62 & 1,31 \\
\hline $\begin{array}{l}\text { Valorização das lideranças } \\
\text { intermédias }\end{array}$ & 1,60 & 1,28 \\
\hline Continuidade da autoavaliação & 1,58 & 1,22 \\
\hline $\begin{array}{l}\text { Adoção de medidas de melhoria } \\
\text { em articulação com a autoavaliação } \\
\text { (...) }\end{array}$ & 1,58 & 1,28 \\
\hline $\begin{array}{l}\text { Promoção do desenvolvimento } \\
\text { profissional dos professores }\end{array}$ & 1,19 & 1,23 \\
\hline Eficácia da comunicação externa & 1,17 & 1,13 \\
\hline $\begin{array}{l}\text { Promoção do desenvolvimento } \\
\text { profissional dos funcionários não } \\
\text { docentes }\end{array}$ & 1,12 & 1,19 \\
\hline $\begin{array}{l}\text { Gestão do desempenho e } \\
\text { competências dos funcionários não } \\
\text { docentes (p. ex., avaliação, } \\
\text { planeamento da formação) }\end{array}$ & 1,10 & 1,14 \\
\hline $\begin{array}{l}\text { Criação de estruturas e/ou práticas } \\
\text { de acompanhamento do percurso } \\
\text { pós-escolar dos alunos }\end{array}$ & 1,02 & 1,19 \\
\hline
\end{tabular}

\section{Loci}

Na parte do instrumento referente aos Loci do impacto da AEE, pedia-se ao respondente que avaliasse numa escala de 5 pontos, entre 0 (Nenhum impacto) e 4 (Impacto muito grande), a magnitude do impacto da AEE em diversos elementos e atores escolares.

Os diretores referem um impacto moderadamente positivo da AEE sobre todos os Loci, embora se destaquem positivamente o impacto sobre a direção, documentos orientadores e órgãos de gestão intermédia, e menos positivamente o pacto sobre entidades externas à escola, pais e recursos disponíveis (Tabela 4).
Tabela 4.

Loci do impacto da AEE

\begin{tabular}{|c|c|c|}
\hline Itens & Méd. & DP \\
\hline Formas de atuação da Direção & 2,67 & 1,18 \\
\hline Documentos orientadores da & 2,65 & 1,13 \\
\hline $\begin{array}{l}\text { atividade da escola/agrupamento (p. } \\
\text { ex., Projeto Educativo, Projetos }\end{array}$ & & \\
\hline Curriculares, Planos de Atividades) & & \\
\hline $\begin{array}{l}\text { Formas de atuação dos órgãos de } \\
\text { gestão intermédia }\end{array}$ & 2,54 & 1,07 \\
\hline Regulamentos internos & 2,40 & 1,10 \\
\hline Formas de atuação dos professores & 2,40 & 1,08 \\
\hline Formas de atuação dos & 2,17 & 1,15 \\
\hline funcionários administrativos & & \\
\hline $\begin{array}{l}\text { Formas de atuação dos auxiliares } \\
\text { de ação educativa }\end{array}$ & 2,13 & 1,10 \\
\hline Formas de atuação dos alunos & 1,92 & 1,15 \\
\hline $\begin{array}{l}\text { Formas de atuação de entidades } \\
\text { externas relevantes }\end{array}$ & 1,90 & 1,19 \\
\hline Formas de atuação dos pais & 1,88 & 1,16 \\
\hline $\begin{array}{l}\text { Materiais e recursos educativos } \\
\text { disponíveis }\end{array}$ & 1,77 & 1,24 \\
\hline
\end{tabular}

\section{Fatores potenciadores e inibidores dos efeitos da AEE}

No bloco seguinte do inquérito, pedia-se aos diretores que apreciassem a influência de determinados fatores como inibidores ou potenciadores do impacto da AEE, numa escala de 5 pontos, que variava entre -2 (efeito muito inibidor) e +2 (efeito muito potenciador).

Verificou-se uma apreciação moderadamente positiva de todos os fatores apontados, dos quais destacamos, na Tabela 5, os 6 fatores cujo impacto foi avaliado como maior, e que se relacionam com o processo de avaliação interna, a difusão dos resultados da AEE, participação dos diferentes atores no processo e apropriação do referencial da IGEC.

Tabela 5.

Fatores potenciadores do impacto da AEE

\begin{tabular}{|c|c|c|}
\hline Itens & Média & SD \\
\hline $\begin{array}{ll}\text { Consequências } & \mathrm{da}\end{array}$ & 1,05 & ,999 \\
\hline $\begin{array}{ll}\text { externa no processo } & \text { de } \\
\text { autoavaliação/equipa } & \text { de } \\
\text { autoavaliação } & \end{array}$ & & \\
\hline $\begin{array}{l}\text { Grau de articulação entre os } \\
\text { processos de avaliação interna e } \\
\text { externa }\end{array}$ & 0,92 & ,860 \\
\hline $\begin{array}{l}\text { Difusão na escola/agrupamento dos } \\
\text { resultados da avaliação externa }\end{array}$ & 0,90 & 913, \\
\hline $\begin{array}{l}\text { Publicitação do relatório de } \\
\text { avaliação externa }\end{array}$ & 0,90 & 891 \\
\hline $\begin{array}{l}\text { Nível de participação/envolvimento } \\
\text { dos diferentes atores da escola no } \\
\text { processo de avaliação }\end{array}$ & 0,81 & 886 \\
\hline $\begin{array}{l}\text { Apropriação e uso pela escola dos } \\
\text { referenciais da avaliação externa (da } \\
\text { IGEC) na sua avaliação e } \\
\text { monitorização interna }\end{array}$ & 0,77 & 1,113 \\
\hline
\end{tabular}

\section{Avaliação global do processo de AEE}

No penúltimo bloco de questões, os diretores expressaram as suas avaliações globais sobre o impacto global da AEE sobre 5 aspetos, recorrendo a uma escala 
de 7 pontos, que variava entre -3 (Extremamente negativo) e +3 (Extremamente positivo). A apreciação global da AEE foi novamente moderadamente positiva, sobre todos os aspetos propostos, embora mais expressivamente no tocante à autoavaliação, e menos expressivamente sobre os resultados dos alunos (Tabela 6).

\begin{tabular}{ccc} 
Itens & Média & SD \\
\hline $\begin{array}{c}\text { Ao nível do processo de } \\
\text { autoavaliação da escola }\end{array}$ & 1,73 & 1,300 \\
$\quad \begin{array}{c}\text { Ao nível organizacional } \\
\text { Ao nível curricular e pedagógico }\end{array}$ & 1,40 & 1,332 \\
$\quad \begin{array}{c}\text { Ao nível da participação da } \\
\text { comunidade na vida da escola }\end{array}$ & 1,12 & 1,293 \\
$\quad$ Ao nível dos resultados dos alunos & 0,92 & 1,218 \\
\hline
\end{tabular}

\section{Eficiência do processo de AEE}

Por fim, pediu-se aos diretores que se pronunciassem numa escala de 5 pontos quanto à eficiência do processo de AEE. A maioria dos respondentes considerou que este processo corresponde a um bom $(n=29,55,8 \%)$ ou moderadamente eficiente $(n=14,26,9 \%)$ aproveitamento de recursos.

\section{Discussão e reflexão conclusiva}

Os resultados obtidos pelas escolas da amostra no processo de AEE foram globalmente positivos e revelam uma melhoria entre ciclos, destacando-se o domínio da Liderança e Gestão.

Os resultados do questionário apontam para uma perceção moderadamente positiva dos impactos e efeitos da avaliação externa de escolas por parte dos diretores. Esta perceção é mais expressiva em relação aos aspetos relacionados com a autoavaliação e a gestão escolar, e menos expressiva em relação aos aspetos que se relacionam mais diretamente com os comportamentos dos alunos e de elementos exteriores à comunidade educativa.

Concretamente, quanto ao domínio Resultados, destacaram-se aspetos relacionados com a participação de diversos elementos da comunidade na escola, o que corresponde a um dos objetivos da AEE, bem como a avaliação interna dos resultados dos alunos. No entanto, as aprendizagens efetivas dos alunos, o seu comportamento e avaliação externa são percebidos como menos influenciáveis pela AEE, o que questiona o seu efeito a nível de um dos seus objetivos essenciais.

No domínio Prestação do Serviço Educativo, parece haver uma tendência para focalizar os resultados e as práticas potenciadoras do sucesso, em detrimento de outros aspetos importantes, como a equidade, o que poderá indiciar algum efeito de «Visão em Túnel» (Ehren \& Visscher, 2006).

No tocante à Gestão e Liderança, a autoavaliação é destacada pelos diretores como um aspeto muito influenciado pela AEE. Esta é também perspetivada como o mais influente fator potenciador do impacto da AEE e merece por isso especial atenção.

Relativamente aos Loci, os Diretores avaliam o impacto tanto mais positivamente, quanto mais nos aproximamos da gestão de topo, decrescendo à medida que nos afastamos desta - professores, alunos, pais, comunidade, recursos e instalações escolares, etc.

Concluímos assim que a avaliação que os diretores das escolas públicas desta região fazem sobre a avaliação externa indica contributos para repensar processos, sobretudo os mais abrangentes ao nível da organização, percebendo no entanto efeitos menos expressivos no que concerne ao trabalho que se desenvolve no interior da sala de aula, ao comportamento dos alunos e dos elementos da comunidade mais alargada. Ao mesmo tempo, a perceção globalmente positiva ilustra o forte potencial da AEE enquanto instrumento de regulação, prestação de contas e melhoria das práticas, que não deverá ser negligenciado.

\section{Referências}

Almeida, L., \& Freire, T. (2008). Metodologia da Investigação em Psicologia e Educação (5. ${ }^{\mathrm{a}}$ Ed.). Braga: Psiquilíbrios.

Ehren, M. C. M., \& Visscher, A. J. (2006). Towards a theory on the impact of schools inspection. British Journal of Educational Studies, 54(1), 51-72.

IGEC (2011). Quadro de referência para a Avaliação Externa das Escolas. Disponível em: http://www.ige.min-edu.pt/upload/AEE_2014-20 15/AEE_14-15_\%281\%29_Quadro_de_Referencia.pd $\mathrm{f}$

Moreira, J. M. (2007). Investigação quantitativa: fundamentos e práticas. In J. Á. Lima \& J. A. Pacheco (Org.), Fazer investigação (pp. 41-84). Porto: Porto Editora.

Moreira, J., \& Rodrigues, P. (2015). Processos e impactos da AEEENS: resultados do inquérito nacional do projeto AEEENS aos diretores de agrupamentos de escolas. In. A. Mouraz, S. Valadas, \& J. A. Pacheco (Orgs.), Avaliação Externa de Escolas do Ensino Não Superior: Coordenadas e processos de um projeto de investigação (pp. 474-482). Porto: CIIE.

Pacheco, J. A., Seabra, F., \& Morgado, J. C. (2014). Avaliação Externa. Para a referencialização de um quadro teórico sobre o impacto e efeitos nas escolas do ensino não superior. In. J. A. Pacheco (Org.). Avaliação Externa de Escolas: Quadro Teórico Conceptual. Porto: Porto Editora.

Rodrigues, E., Seabra, F., Queirós, H., Sousa, J., Lamela, C., Costa, N., \& Morgado, J. C. (2015, no prelo). Avaliação externa e seus efeitos: perspetivas dos atores escolares. In. C. Barreira (Org.). Estudos sobre avaliação externa de escolas. Lisboa: Porto Editora.

Rodrigues, P. \& Moreira, J. (2015). O questionário na avaliação de escolas: o caso do impacto da avaliação. In. A. Mouraz, S. Valadas, \& J. A. Pacheco (Orgs.), Avaliação Externa de Escolas do Ensino Não Superior: Coordenadas e processos de um projeto de investigação (pp. 97-101). Porto: CIIE.

Tuckman, B. (2000). Manual de investigação em Educação. Lisboa: Fundação Calouste Gulbenkian. 